\title{
Effects of Radiation on Mitochondrial Defective Lymphoblastoid Cells
}

\author{
Jessica Chen ${ }^{1}$, Deepa Sridharan ${ }^{1}$, Chad Cross ${ }^{2}$ and Janice Pluth ${ }^{2 *}$ \\ ${ }^{1}$ UC Berkeley; Berkeley, CA, USA \\ ${ }^{2}$ University of Nevada, Las Vegas, USA \\ *Corresponding author: Janice Pluth, University of Nevada, Las Vegas, Maryland Parkway, Las Vegas, NV, USA; E-mail: janice.pluth@unlv.edu
}

Received: July 27, 2021; Accepted: August 27, 2021; Published: November 27, 2021

\begin{abstract}
Background: Ionizing radiation results in an increase in ROS and can be further enhanced in cells with damaged mitochondria, inducing persistent damage and genomic instability, leading to cancer. DNA damage response (DDR) is activated post radiation, and combined with ROS can result in telomere length changes. Mitochondria play a key role in mediating non-targeted effects post radiation exposure. Mitochondrial DNA (mtDNA), due to its less efficient repair as compared to nuclear DNA, is more severely affected by radiation exposure and mutations in essential mtDNA genes can further exacerbate oxidative stress.
\end{abstract}

Aim: We evaluated the impact of mitochondrial defects on radiation-induced DDR and telomere length changes, biomarkers that can foreshadow cancer development.

Methods: To better understand this relationship, we have used lymphocytes containing various mitochondrial mutations and studied the kinetics of DNA damage and telomere length changes over time post radiation exposure. In this study we investigated the kinetics of DNA damage response and telomere length changes in cells in lymphoblastoid cell lines with known mitochondrial mutations following a 0.5 Gy dose of Xray or $1 \mathrm{GeV} / \mathrm{um}$ Fe ion.

Results: Our studies suggest that cells containing different mitochondrial mutations have unique DNA damage and telomere length effects following radiation exposure. The effects on telomere length also reveal differences dependent upon the radiation quality of exposure. Results revealed cells with a mitochondrial mutation in the ND4 subunit of complex 1 showed a decreased growth rate, higher levels of persistent DNA damage, and telomere instability as compared to wild type. In contrast the ATPase 6 mitochondrial mutant showed more subtle changes.

Conclusion: These findings point to the importance of mitochondrial integrity and the role it may play in cellular changes that promote cancer. In total results indicate mitochondrial mutations can influence DNA damage kinetics and telomere length and have long-term consequences in regaining homeostasis following radiation exposure. These results may aid in understanding the rationale for the persistent genomic instability following a low dose of radiation and changes leading to cancer promotion.

Keywords: DNA Damage, Mitochondria, Radiation, Telomere

\section{Introduction}

Ionizing radiation is believed to cause changes within cells that lead to either the initiation and/or promotion of cancer. Although previous studies have focused mainly on mutations as the primary culprit in increasing cancer risk, non-targeted effects are also thought to impact cancer risk [1]. Reactive oxygen species (ROS) are induced by ionizing radiation and can persist for long time periods following radiation [2], perhaps playing a role in non-targeted effects that lead to cancer development.

ROS are produced by water radiolysis following ionizing radiation exposure, and generated more predominantly by $\gamma$-rays and X-ray (low linear energy transfer, or LET, radiation) as compared to high LET radiation, which causes more direct effects [3]. Unrepaired mitochondrial and nuclear DNA damage is thought to further increase ROS levels and increase oxidative stress within the cells [4] and thus increases in ROS have been linked to higher levels of DNA damage
[5]. Mitochondrial mutations can result in a chronic production of superoxide radicals within the cytoplasm [6]. This is especially important given the DNA within the mitochondria is less efficiently repaired [7], and thus at a greater risk to the effects of radiation. In addition, mtDNA mutations have been associated with aging and cancer [8-10].

Early studies out of the Little laboratory were some of the first to note that cells may exhibit damage following radiation that is not from direct exposure. In these studies, they noted an increase in sister chromatid exchanges which were much higher than expected given the low dose exposure the cells received [11]. This elevated level also lasted for many generations following the exposure, indicating a persistent nature of the insult. Since this time additional studies investigating the nature of this damage have been initiated and more recent work has revealed an important role for the mitochondria in non-targeted effects (NTE) within cells following radiation [12]. In 
this latter work they noted that irradiated cells released mtDNA into vesicles that acted as signals to bystander cells, whereas irradiated cells lacking mtDNA did not show an increase in DNA damage levels in un-irradiated bystander cells. This work strengthens the notion that mtDNA play important roles within the cell and influence the DNA damage response in a non-targeted manner.

Mitochondria have essential roles within cells providing energy and generating ATP [13]. Established lymphoblastoid cell lines containing mitochondrial mutations have been used to study how radiation effects may differ in cells with defective mitochondria $[14,15]$. One such line, from a patient with Leigh's syndrome, contains a mutation in a region of the mtDNA that encodes for the ATP6 gene, a part of complex V in ATP synthesis [16]. Another cell line is from a patient with Leber's optic atrophy and has a mutation in the ND4 gene, a component of complex I in the ATP synthesis machinery [17]. A previous study by Kulkarni et al., [15] noted that cell lines with mitochondrial mutations responded uniquely to ionizing radiation as compared to normal human lymphoblastoid cells, showing increased chromosomal aberrations and reduced mitotic indices. Further subsequent work from this same group [14] also revealed differences in mitochondrial gene expression over time post radiation exposure and suggested that people with these mitochondrial mutations are likely sensitive to mutagens and that caution should be used if they are provided radiation therapy.

In this study we were interested in better understanding the contribution defective mitochondria have on the biological effects observed post a dose of radiation which most normal cells can easily repair damages $(0.5 \mathrm{~Gy})$. To test the impact of radiation on cells containing defective mitochondria, we assayed DNA damage foci and telomere length kinetics over time, focusing on persistent and late effects (weeks post exposure), in lymphoblastoid cells with and without known mitochondrial defects. Much later time points were focused on due to the link between persistent longer-term DDR signaling and cancer [18]. Additionally, given that differential effects may be expected following low vs high LET radiation exposure, we also assayed telomere length changes in these cell lines exposed to both radiation qualities. In total, our findings implicate the mitochondria as playing an important role in the biological effects observed, and suggest that particular mitochondrial defects result in more or less severe outcomes.

\section{Methods}

\section{Cell Lines}

All lymphoblastoid cell lines were acquired from Coriell cell repositories (Camden, NJ) and passaged to produce enough frozen down stocks of similar passage for these experiments. GM15510 and GM15036 are normal human lymphoblastoid cell lines without known mutations, whereas GM13740 and GM10744 are cell lines containing mitochondrial mutations. GM13740 was obtained from a male subject 12 years of age, diagnosed with Leigh Syndrome. GM13740 contains a T to G transversion at the nucleotide pair 8993, resulting in a leucine to arginine at position 156 in subunit 6 of the mitochondrial $\mathrm{H}(+)$-ATPase. GM10744 is from a male 53-year-old subject diagnosed with Leber Optic Atrophy, and contains a causing a guanine to adenine transition mtDNA mutation at position 11778 $(11778 \mathrm{G}>\mathrm{A})$ in the NADH dehydrogenase subunit 4 gene (MTND4). All cell lines were cultured in 1640 RPMI medium (Gibco) containing 15\% Fetal bovine serum (Gibco), 1.1\% Antibiotic-antimitotic (Gibco) and $2 \mathrm{mM} \mathrm{L-glutamine} \mathrm{(Gibco).} \mathrm{Cell} \mathrm{culture} \mathrm{flasks} \mathrm{were} \mathrm{kept} \mathrm{in} \mathrm{a}$ humidified incubator at $37^{\circ} \mathrm{C}$ and at $5 \% \mathrm{CO}_{2}$ concentration.

\section{Radiation Exposures}

Cells were seeded at $1 \times 10^{6}$ cells $/ \mathrm{mL}$ in $8 \mathrm{~mL}$ of culture medium and placed into T25 vented flasks (Corning) for each timepoint acquisition and incubated overnight. Cells were then exposed to $0 \mathrm{~Gy}$ (Control) and $0.5 \mathrm{~Gy}$ of X-ray at a dose rate of approximately $0.55 \mathrm{~Gy} /$ min using a Faxitron X-Ray Source. Once irradiated, the cells were returned to the incubator and harvested on days $1,3,8,10,13$, and 16 days (depending upon the endpoint, not all days were each endpoint collected, see figures for specifics) post X-Ray or Fe ion exposure. For $1 \mathrm{GeV} / \mathrm{um}$ Fe ion exposure NASA's Space Radiation Laboratory (NSRL) was used. Fe ion exposure was at a dose rate of approximately $23.52 \mathrm{cGy} / \mathrm{min}$. NSRL uses heavy ions from Brookhaven National Laboratory's (Upton, NY) booster accelerator to produce the various ion beams used for testing cellular effects to space radiation.

\section{Growth Curve}

Cells were counted and set up in multiple T25 flasks, and total numbers of cells were counted on various days following this initial set up. Half the flasks for each line were irradiated to define effects of 0.5 Gy dose of X-ray on growth. Separate flasks were set up in duplicate to count on days $1,3,8,10,13$, and 16 post $\mathrm{X}$-ray or mock irradiation. Flasks for future cell counts were all fed on days which cell counts were performed. To count cells, cells were removed from flasks, spun down, supernatant removed and trypan blue used to define $\%$ viability (typically between $75-90 \%$ for all lines) on each day counts were taken. Cells from a portion of the culture were also counted on a coulter counter to define absolute numbers of cells within the culture. The average number of total cells on each day with SEM is shown in Figure 1. The population doublings were calculated during exponential growth from counts on Days 3 and 10 and compiled in Table 1.

\section{Gh2ax Foci Staining and Analysis}

On days 1, 3, and 13 post X-ray exposure cells were plated onto poly-l-lysine coated chamber slides (Lab Tek), fixed twice with $1.5 \%$ PFA for 10 minutes at room temperature, followed by a $100 \%$ methanol fixation for 10 minutes at $4^{\circ} \mathrm{C}$. Within a week post fixation, slides were subsequently stained for $\gamma \mathrm{H} 2 \mathrm{AX}$. Primary and secondary antibodies were diluted 1:1 in a $1 \%$ BSA/Odyssey Buffer (LI-COR,

Table 1: Days required for one population doubling for each lymphoblastoid line.

\begin{tabular}{|l|c|c|}
\hline Cell Line & 0 Gy & 0.5 Gy \\
\hline GM15510 & 2.47 & 3.70 \\
\hline GM15036 & 2.67 & 3.56 \\
\hline GM13740 & 2.98 & 4.45 \\
\hline GM10744 & 3.21 & 5.69 \\
\hline
\end{tabular}


Lincoln, NE) mixture. Cells were blocked with 1\% BSA for one hour and then incubated with an anti-phospho-histone H2A.X (Ser139) antibody, clone JBW301, at a dilution of 1:600 (Millipore, Sigma, Burlington, MA). Wells were washed three times for 10 minutes each with a $1: 1$ solution of $1 \%$ BSA in PBS and Odyssey Buffer mixture, and then incubated with a secondary antibody (1:800) Alexa Fluor 594 goat anti-rabbit (Life Technologies, Carlsbad, CA). Cells were washed two times in PBS for 10 minutes each before counterstaining with 300 nM DAPI. Cells were mounted with Vectashield Mounting Medium (Vector Laboratories, Burlingame, CA) and imaged using a Nikon Eclipse TE2000 Inverted microscope with a 40X Objective. Two independent experiments were performed a total of at least 400 cells were scored for each cell line and treatment group to define numbers of gH2AX radiation induced foci (RIF). In a few cases we were unable to score 400 cells, and in these cases between 130-275 cells were scored.

\section{Telomere Length Measurements}

\section{The Flow-FISH Assay for Quantifying Telomere Length}

The Telomere PNA (peptide nucleic acid) Flow-FISH/ FITC kit (Agilent, Santa Clara, CA) was used to examine telomere length on days 3 and 16 post X-Ray or Fe ion exposure. Briefly, cells were counted and $1 \times 10^{6}$ cells frozen down in $80 \% \mathrm{FBS} / 20 \%$ DMSO on each day of collection and kept at $-80^{\circ} \mathrm{C}$ until further analysis. Vials were thawed at $37^{\circ} \mathrm{C}$, brought up in medium containing $10 \%$ serum and washed in PBS. Each sample was divided into four aliquots and placed into $1.5 \mathrm{ml}$ Eppendorf tubes and labeled A-D. Samples were centrifuged at $500 \mathrm{x} \mathrm{g}$ and supernatant removed. $300 \mathrm{ul}$ of hybridization solution was added to 2 of the tubes and $300 \mathrm{ul}$ of telomere PNA/Probe/FITC in Hybridization solution was added to the other 2 tubes. Lids were closed and tubes mixed by vortexing. The tubes were placed on a prewarmed heating block adjusted to $82^{\circ} \mathrm{C}$. Tubes were removed after 10 minutes and again mixed using the vortex and placed in the dark at room temperature overnight. The following day $1 \mathrm{ml}$ of $1 \mathrm{X}$ wash solution was added to each of the 4 tubes and vortexed. The tubes were then placed on a pre-warmed heating block at $40^{\circ} \mathrm{C}$ for 10 minutes. Samples were then mixed on the vortex and centrifuged at $500 \mathrm{x} \mathrm{g}$. This was repeated a second time following removal of the supernatant and a DNA staining solution (containing RNAse and PI) was then added and samples were left at $2-8^{\circ} \mathrm{C}$ for 2-3 hours prior to analysis on a BD FACSCalibur flow cytometer.

\section{Analysis of Flow Cytometry Files}

Samples were analyzed using a logarithmic scale FL1-H for FITC probe (levels of FITC corresponding to lengths of telomere) and on linear scale FL3-H for DNA staining. Samples were analyzed using Flowjo software (Becton Dickinson) and strategy for gating is noted in Figure 2. For analysis, the average fluorescent levels (corresponding to the length of the telomere) for each sample are compared to its nonirradiated control level of fluorescence.

\section{Statistical Analysis}

To examine potential cell line differences for telomere length changes and for the percentage of cells with $>3$ foci, we utilized a resampling statistical procedure to construct a bootstrapped sampling distribution of the difference between values for each comparison. Each bootstrap distribution was constructed from 10,000 resamples, which were then used to construct a $95 \%$ confidence interval of the resulting differences. Using this method, and under a null of no difference, confidence intervals not containing a zero difference are significant at $\mathrm{p}<0.05$. We utilized the program $\mathrm{R}$ bootstrapping routine (as implemented in artofstat.com/web-apps).

\section{Results}

\section{Growth of Lymphoblastoid Lines}

The growth rate plus and minus $0.5 \mathrm{~Gy} \mathrm{X}$-ray was defined for each lymphoblastiod line (Figure 1). Cell counts were defined on days 1-16 after having seeded $5 \times 10^{6}$ cells on day 0 . Duplicate samples were set up and counted each day using a coulter counter

(Beckman Coulter, Indianapolis, IN). All lines grew fairly similarly both plus and minus radiation with the exception of one of the mitochondrial mutant lines, GM10744,

appeared to slightly decrease the growth rate for all cell lines. When viewing population doubling times as calculated during exponential growth (Day 3 to Day 10) for each line (Table 1) the mitochondrial mutant lines showed a slightly higher number of days needed for a population doubling as compared to the normal wild type lines, both with and without radiation.

\section{Gh2ax Foci Kinetics}

The kinetics of $\gamma \mathrm{H} 2 \mathrm{AX}$ radiation induced foci (RIF) were defined over time post $0.5 \mathrm{~Gy}$ X-ray. $\gamma \mathrm{H} 2 \mathrm{AX}$ are generally regarded to be a marker a marker of DNA double strand breaks (DSB) [19], and the activation of the DNA damage response (DDR) has been shown to be an important element in the progression of cancers [2,20]. Levels of RIF were binned based on the number of foci per cell and the number of cells containing greater than 3 foci quantified for days 1, 3 and 10 post X-ray exposure (Figure 3 ). which grew at a lower rate as compared to the other lines. In comparing each line's growth following $0.5 \mathrm{~Gy} \mathrm{X}$-ray to the same line without radiation $(\mathrm{C}-\mathrm{F})$, radiation

Normal control cells can contain lower numbers of RIF in the process of normal growth, so to better distinguish cells that were more heavily affected by the radiation exposure we centered on cells with at least 3 RIF. A representative image of cells showing various binned categories of foci is shown in Figure 3A. Our results show fairly similar levels of foci in control non-irradiated cells for all cell lines. Day 1 post radiation maximal levels were observed for all lines (Figure 3B), with the mitochondrial defective line (GM10744) showing the greatest induction. Levels of RIF decreased for wild type cells in subsequent days but remained elevated over control for the two mitochondrial defective lines. On day 10 post radiation, we observe that both GM15510 and GM15536 returned to per-radiation levels. Only the mitochondrial defective lines GM13740 and GM10744 showed residual foci above control levels. This persistent DDR would predict that these cell lines would have a greater potential of becoming genomically unstable and having a greater risk of becoming transformed. 
A

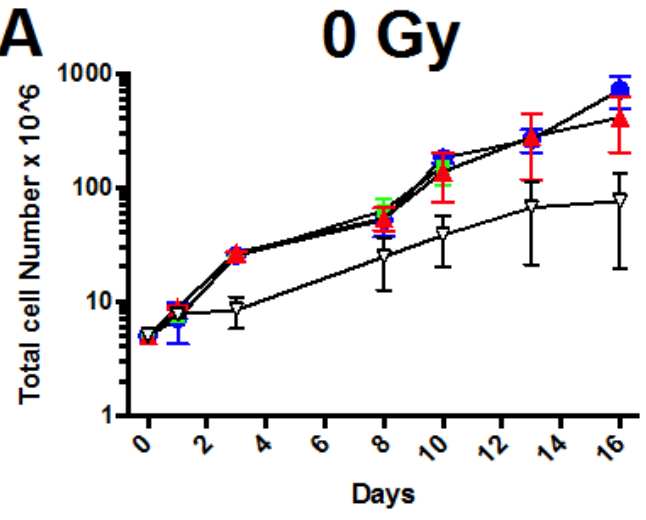

C
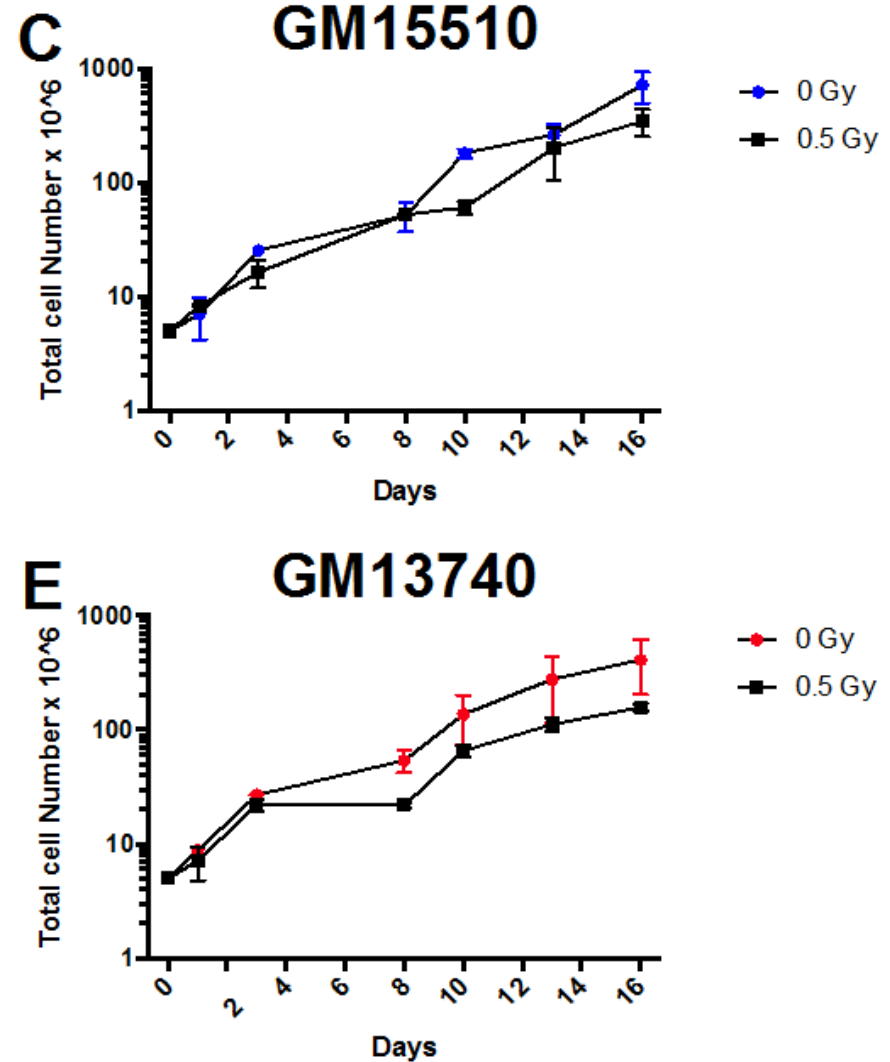

$\rightarrow 0.5 \mathrm{~Gy}$
B

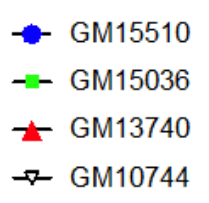

$\rightarrow 0 \mathrm{~Gy}$

D
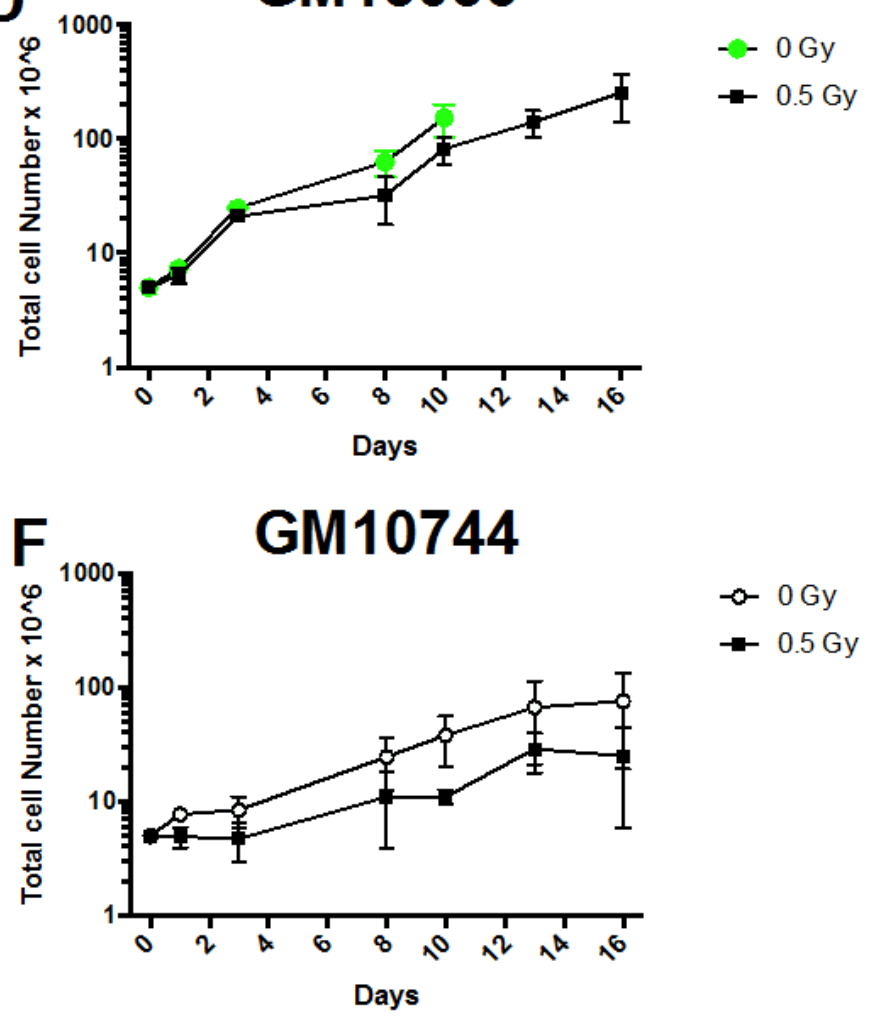

Figure 1: Growth curves for wild type (GM15510 and GM15536) and mitochondrial defective (GM13740 and GM13744) lymphoblastoid lines. Comparison of growth for lines without radiation (A) and following 0.5 Gy of X-ray (B). The growth for each line is also compared below this for control (0 Gy) and irradiated (0.5 Gy), for GM15510 (C), GM15036 (D), GM13740 (E) and GM10744 (F). Cells were seeded into multi-well plates on day 0 and counted on days 1,3, 8, 10, 13 and 16.
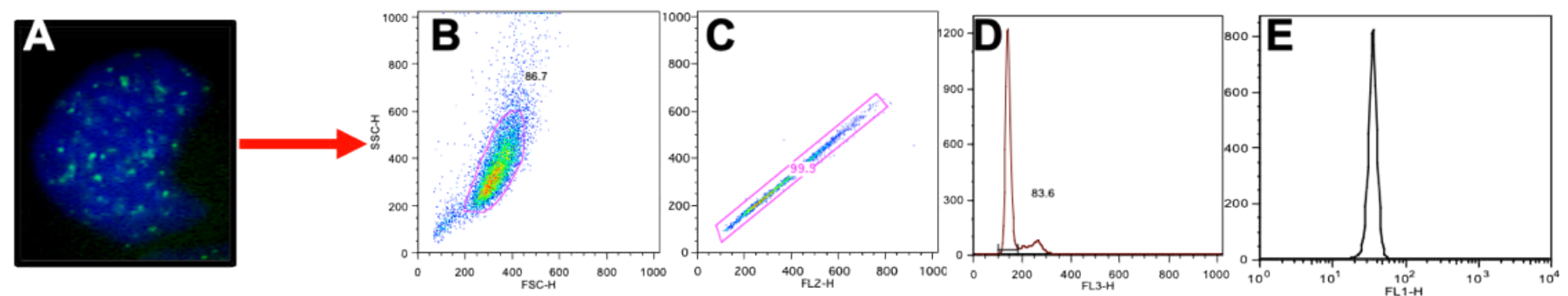

Figure 2: Gating Scheme for telomere analysis. A visual example of a cell stained with PNA telomere probe in (A). Stained cells were analyzed on a BD FACSCaliber and resulting files gated using Flowjo software (BD). Primary cell population was gated on in FSC vs SSC (B) and doublets were eliminated by plotting FL1 (H) vs (W) (C). This gated population was then used to identify the G1 population (D) and the mean FL1 (FITC) fluorescence (E) was then determined based on the total G1 population of cells. Mean FL1 values are compared to controls levels to provide the relative telomere lengths for each cell line and treatment. 

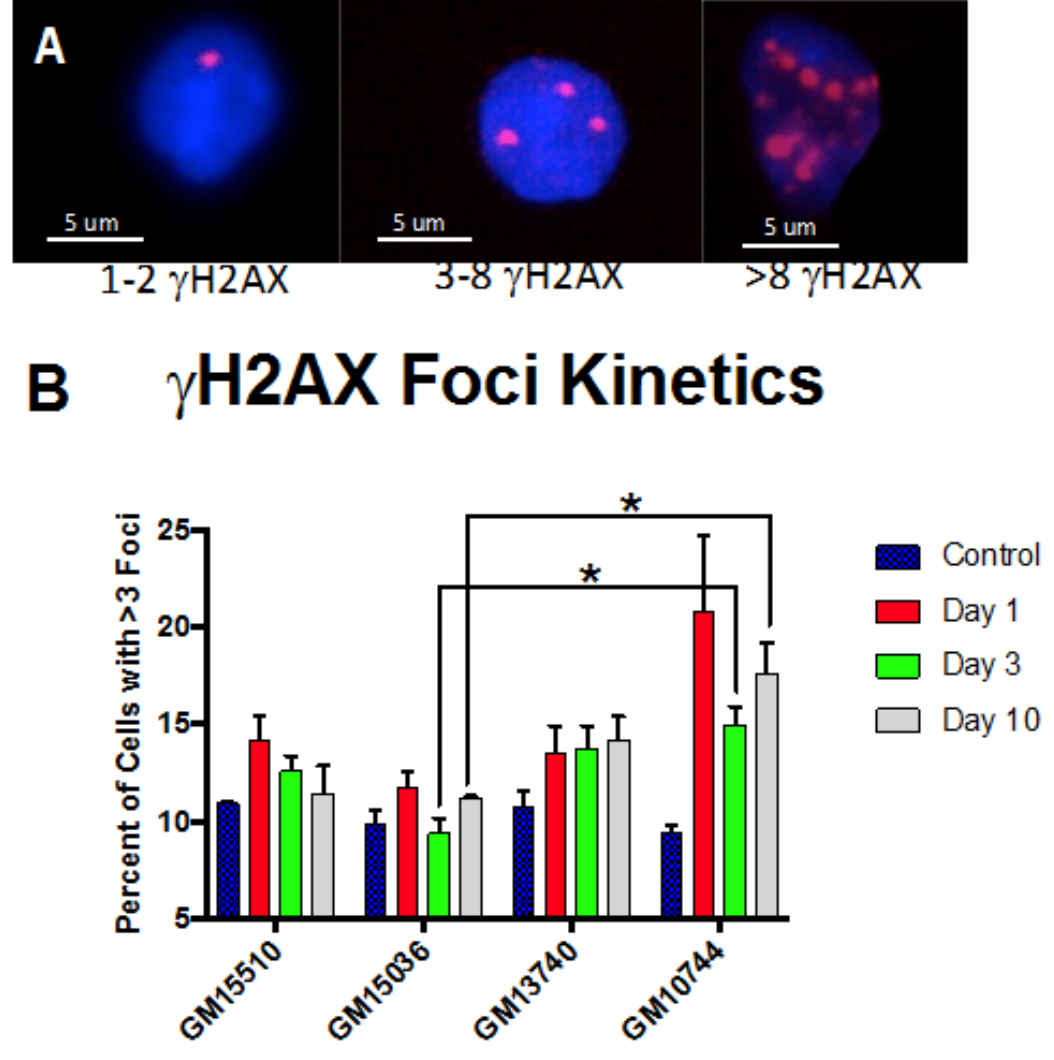

Cell Line

C GM15510

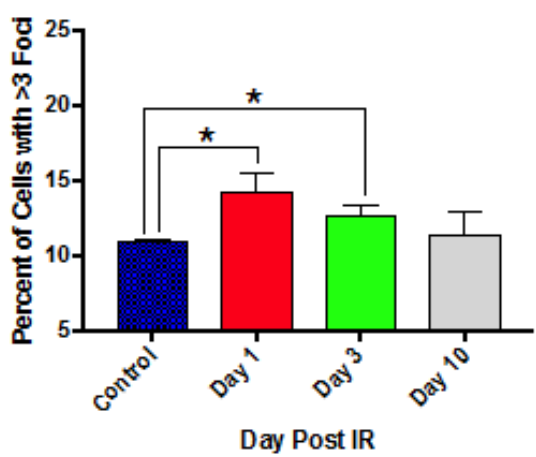

E $\quad$ GM13740

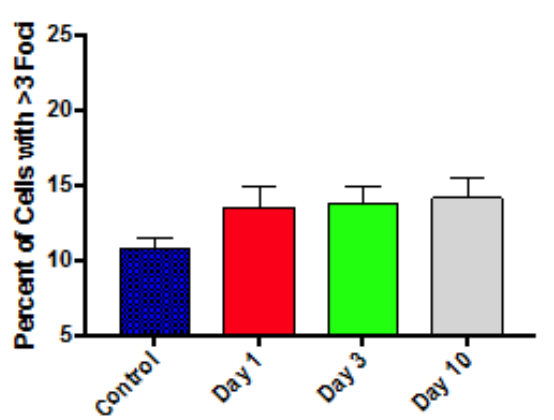

Day Post IR

D
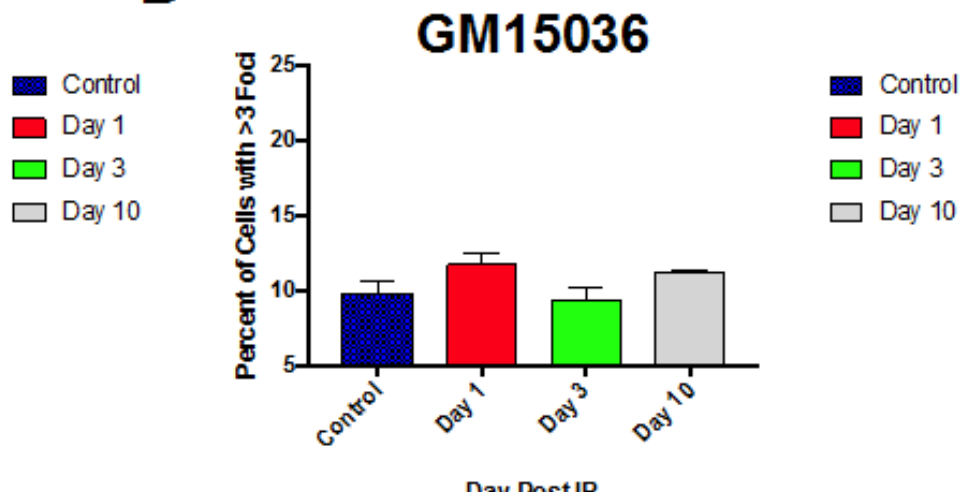

$\mathbf{F}$

Day Post IR

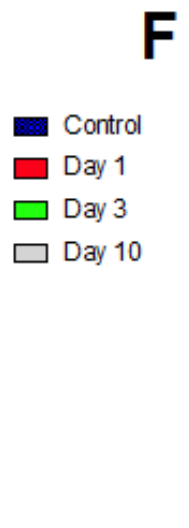

GM10744

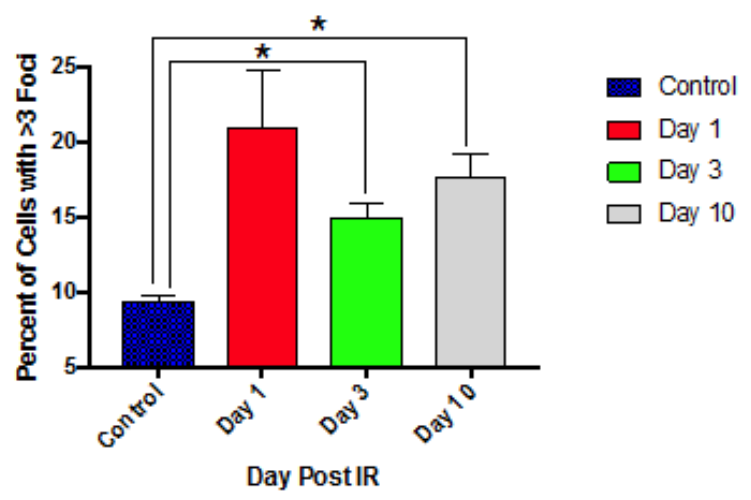

Figure 3: Kinetics of gH2AX foci post 0.5 Gy X-ray. A representative image of cells taken using a 40X objective shows the various categories of foci scored for each cell line, treatment and day (A). The percent of cells with greater than 3 RIF in averaged control samples and on days 1, 3 and 10 post exposure for all lines (B) and for each line separately (C-F). 


\section{Radiation Quality Effects on Telomere Length and Influence} of Mitochondrial Mutations

The change in telomere length following X-ray exposure was also monitored on days 3 and 16 (Figure 4). Relative telomere lengths as compared to controls are plotted. Significant differences are noted on day 3 between the mitochondrial defective line GM10744 and both GM15536 (WT) and GM13740 on day 3. GM10744 was the only line to show a lengthening of the telomere on day 3 which increased further in length on day 16 . On day 16 significant differences were noted between both WT lines, with GM15536 showing a slightly higher average length and GM15510 a slightly lower average telomere length. All lines on day 16 were significantly different from GM10744 which showed a marked increase in average telomere length. Given low LET radiation, like X-ray, is known to produce its more of its effects through indirect action, whereas high LET radiation, like Fe ions, is known to produce more direct effects, we chose to also compare telomere length changes for these lines following a high LET exposure. Following high LET exposure, we did not see the

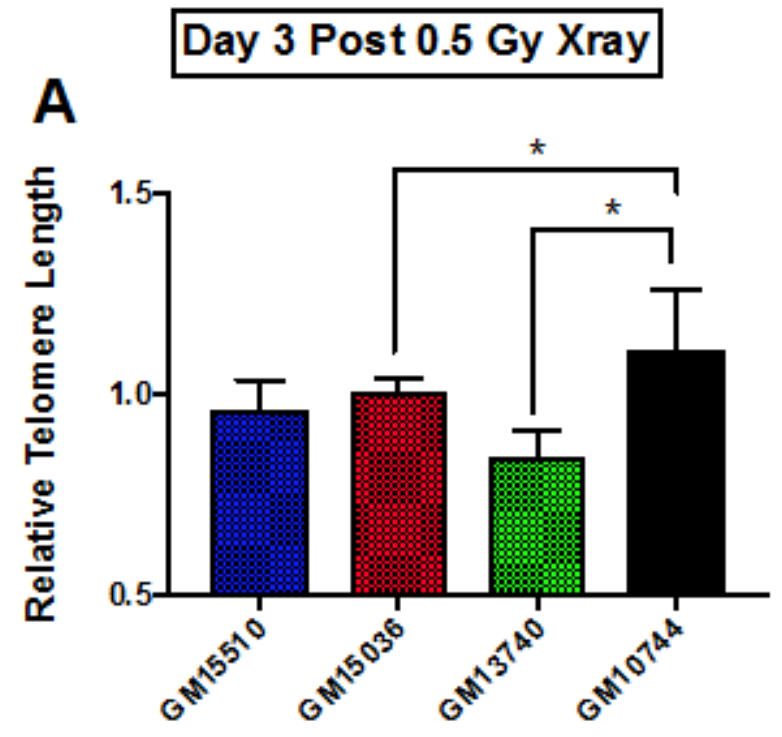

Cell line

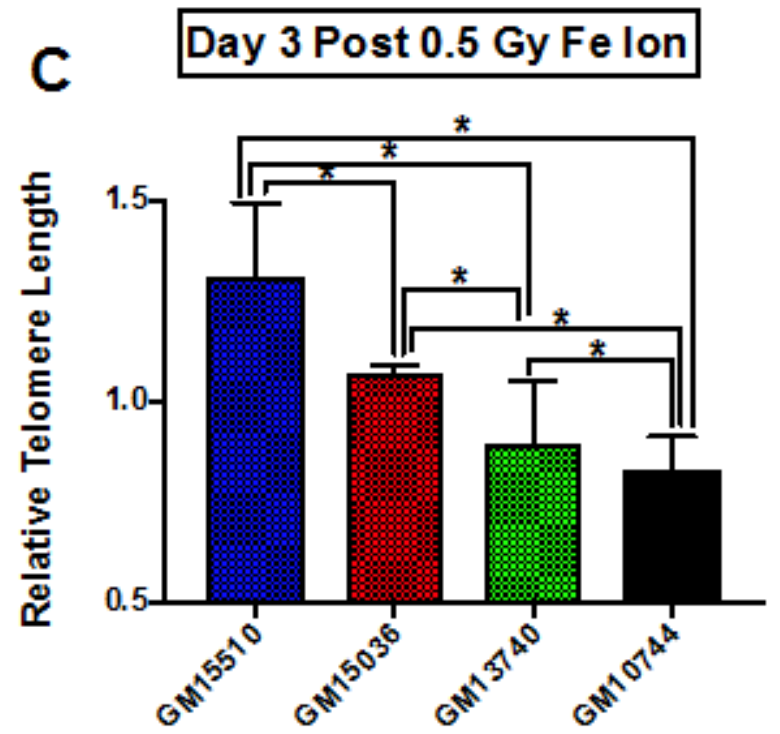

Cell Line

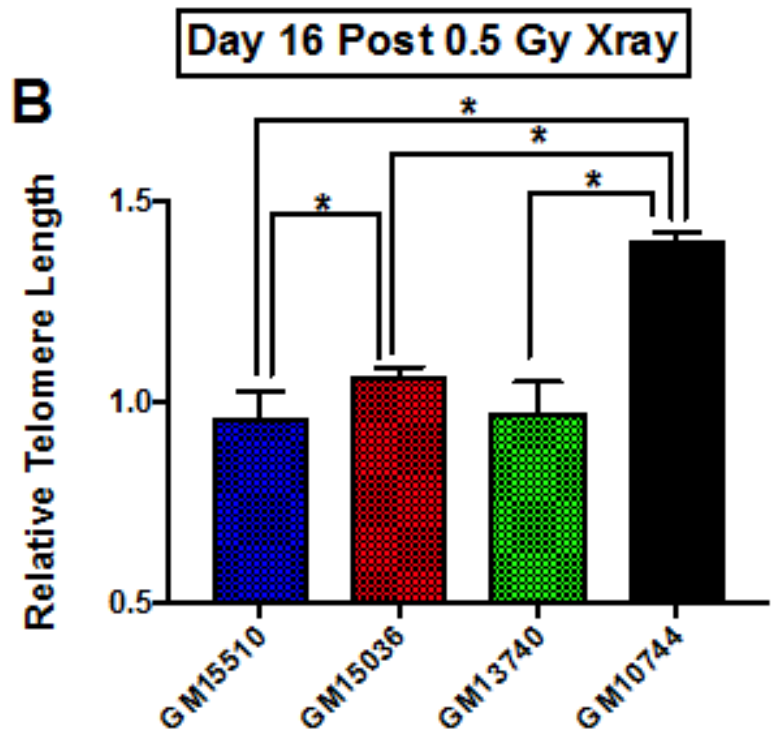

Cell Line

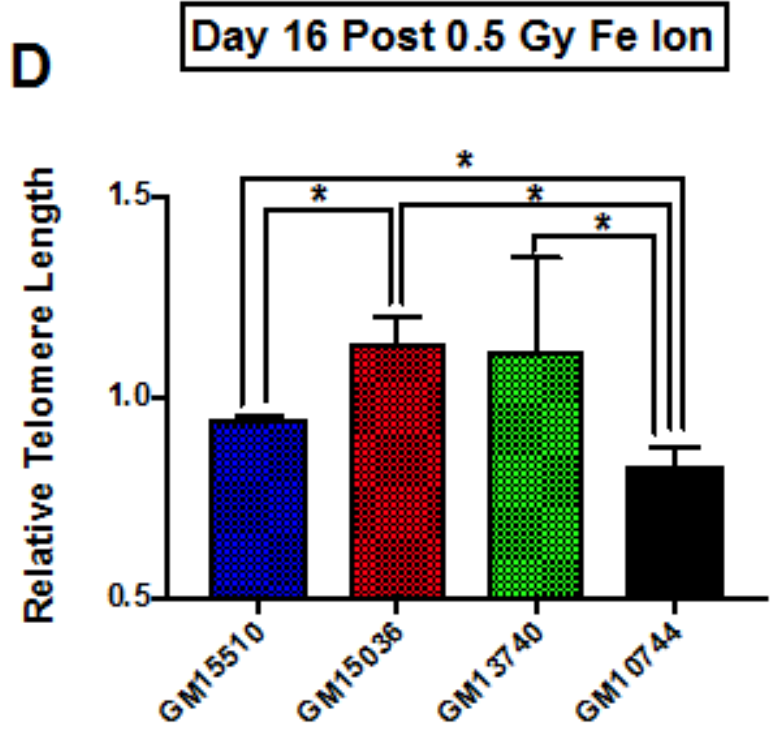

\section{Cell Line}

Figure 4: Relative average telomere lengths for various lymphoblastoid cell lines. Average relative telomere lengths were determined for each cell line on day 3 post X-ray (A), day 16 post X-ray (B), day 3 post $1 \mathrm{GeV} / \mathrm{um}$ Fe ion exposure (C) and day 16 post $1 \mathrm{GeV} / \mathrm{um}$ Fe ion exposure (D). The Y axis is the relative telomere length as compared to control fluorescence levels, thus if equal to control levels it would have a value of 1.0 . 
persistent lengthened telomere phenotype we noted post X-ray but instead a shorter averaged telomere length on both day 3 and 16 for the mitochondrial defective cell line GM10744. All lines showed significant differences with GM10744 on both days. The WT line GM15510 also showed a longer average telomere length on day 3 post Fe ion but then returned to close to base line levels on day 16. It is of interest that opposite effects are observed for the mitochondrial mutant line GM10744 depending on whether the exposure is a result of high or low LET radiation, and that persistent effects are observed and appear to be unresolved even weeks post exposure.

\section{Discussion}

In total, this work suggests that mitochondrial mutations may be important in affecting radiation outcomes and potentially impacting cancer risk. Persistent DNA DSBs have been shown to serve as a biomarker of cancer and was one of the endpoints we chose to assay over time. In addition, telomere length has been associated with cancer risk, thus another endpoint assayed for in this study. We used lymphoblastoid lines with and without known mitochondrial mutations to help shed light on the importance of the mitochondria on the biological outcome following radiation exposure and its effect on cancer risk.

In terms of cell growth, both mitochondrial defective lines revealed slightly delayed growth, exhibiting longer population doubling times, both with and without radiation (Figure 1 and Table 1). In comparing the effects on growth with and without radiation within each cell line, wild type lines showed a decrease of $50 \%$ in population doubling comparing irradiated samples to controls, whereas irradiated mitochondrial defective lines decreased population doubling time by $70 \%$ of control values. Thus, there was a slightly greater effect of radiation on the growth in the mitochondrial mutant cells. Previous work by Kulkarni et al. [14] comparing the normalized cell viability for GM15036, GM13740 and GM10744 using the MTS assay, revealed that GM13740 appeared resistant to radiation as compare to other lines. In addition, GM13740 showed a slight decrease in normalized cell viability as compared to controls. This would indicate that the mitochondrial mutant line GM10744 grows equally well whether irradiated or not and only GM13740 shows a decrease in viability following radiation exposure using the MTS assay. Our results using standard absolute counts did not reveal GM10744 to show any greater resistance to radiation, in fact all lines showing a slight decrease with irradiation. We suspect the differences observed between our results and the work by Kuldarni et al. [14] are due to the assay used and the normalization of viability. Although the slight differences in growth we observed were not a major point of in this paper, future studies verifying these differences using a different method of quantifying the changes would be important to conclusively prove how growth is affected with and without radiation in these mitochondrial cell lines.

Other higher dose gH2AX studies have been done previously by others with these cell lines ( 2 and $4 \mathrm{~Gy}$ ) looking at earlier time points (1-24 h) post exposure [14]. In this previous work they observed that post radiation exposure GM13740 had more breaks than either GM10744 or GM15036 at both 1 and $24 \mathrm{~h}$ timepoints. Whereas GM10744 and GM15036 levels of phosphorylation returned to baseline by $24 \mathrm{~h}$. In our studies evaluating the kinetics of DNA damage response at later time points, to define persistent DDR, we noted that the mitochondrial mutant line, GM10744, revealed the greatest induction as well as the greatest persistence over the period of study (Days 1-10). However significant differences $(\mathrm{p}<0.05)$ were only noted on days 3 and 10, and only between one of the wild type lines, GM15036 and the mitochondrial mutant line, GM10744 (Figure 3B). In comparing levels within each cell line on each day, significant differences were noted in wild type line, GM15510, between control and day 1, and control and day 3. The other wildtype line, GM15036, did not show much of an induction on day 1 and revealed levels fluctuating around control on subsequent days. The mitochondrial mutant line, GM13740, revealed a unique pattern of a slight induction, which did not vary on subsequent days and remained at this persistently elevated state that was not significantly elevated over controls. Lastly, the second mitochondrial mutant line, GM10744, showed maximal phosphorylation levels on day 1 , and some reduction on subsequent days, still showing significant elevation on days 3 and 10 , and the greatest level of persistent DDR for any of the lines. Thus, our results suggest that mitochondrial mutations can impact the level of DNA damage signaling and its persistence, both of which are known risk factors in cancer [7]. Our results at $0.5 \mathrm{~Gy}$ fit well with previous work looking at levels of expression of various DNA repair genes in these cell lines. Following the 0.5 Gy dose the GM13740 line did not show a decrease in the repair genes, whereas the GM13744 line did. At higher doses (1-4 Gy) however, both of these cell lines showed a reduction in DNA repair protein expression ( $\sim 50 \%$ reduction).

Telomeres play important roles in chromosome maintenance and genome stability, and previous studies have suggested a connection between telomere dysfunction and cancer initiation and development [8]. Thus, in the current work we also assayed telomere length for changes post radiation in this cohort of cell lines. Our results at $0.5 \mathrm{~Gy}$ fit well with previous work looking at levels of expression of various DNA repair genes in these cell lines [20]. Following the 0.5 Gy dose the GM13740 line did not show a decrease in the repair genes, whereas the GM13744 line did. At higher doses (1-4 Gy) however, both of these cell lines showed a reduction in DNA repair protein expression ( $\sim 50 \%$ reduction). As high LET radiation is thought to exert its effects through more of a direct, rather than indirect mechanism (that often involves ROS), we also assayed telomere length following a $0.5 \mathrm{~Gy}$ dose of $1 \mathrm{GeV} / \mathrm{um}$ Fe ion exposure. Surprisingly, unique effects were observed following high LET as compared to low LET radiation. The mitochondrial mutant line, GM10744, showed a significantly shorter telomere length than any of the other lines and this shorter length was persistent from day 3 to day 16 . Other differences were also noted in the other cell lines comparing high to low LET radiation, including an early lengthening and delayed lengthen for the wild type lines, GM15510 and GM15036, respectively. GM13740, the other mitochondrial mutant line showed a greater shortening with high LET exposure, which was significantly different from wild type lines on day 3 but then lengthened to wildtype line GM15036 length by day 16 . Thus, all telomeres appear to be more destabilized following the high LET radiation but in particular mitochondrial mutant lines are more severely affected. 
Prior telomere studies have more often seen a link between short telomeres and certain cancers (skin, urogenital, breast, lung, head and neck and lymphoma [21-27]. However, additional published work has indicated various disease states are related to extremes in both short and long telomere lengths, and suggested that long telomeres carried a greater risk of cancer susceptibility than short telomere length in humans [28]. Cancers which have been associated with long telomeres include melanoma [29,30], soft tissue sarcomas [31] and nonHodgkin lymphoma [3]. Thus, both extremes in both long and short telomeres as measured in peripheral blood lymphocytes may increase cancer risk. The rationale for the link between shortened telomeres and cancer risk is that short telomeres are unable to properly cap the ends of their chromosomes and these sticky ends can then fuse to other chromosomes, resulting in genomic instability. However, the molecular mechanisms for telomere lengthening is uncertain, but been speculated that longer telomeres cause an elevated cancer risk as they allow cells to continue to proliferate, evade cellular senescence, and this then allows these cells to accumulate genetic lesions [32]. Future studies would be needed to uncover the mechanism involved and to determine whether additional cancer types are associated with a longer or shorter telomere phenotype and the relationship to radiation quality.

There are at three primary ways that have been used to measure telomere length, and strengths and weakness for each method. In this study we chose to perform flow FISH as previous work had shown it to be more accurate and reproducible as compared to the qPCR method to determine telomere length [33]. Flow-FISH was also shown to have a better agreement with TRF analysis results by Southern blot (typically much more labor-intensive but often regarded as the gold standard) as compared to qPCR [33]. Thus, we believe our study results using flow FISH are accurate but further studies using another means could be useful in validating our results.

Although the work suggests that mitochondrial mutations may impact the DNA damage kinetics and telomere length of the cells following radiation, to better substantiate these findings additional experiments using the GM10744 corrected for this mutation should be studied. Cell lines corrected for this mutation and/or another cell lines with the same mutation in MTND4, would aid in more firmly establishing the importance of mitochondrial mutations and their effect on these processes and their potential link to cancer development.

\section{Conclusion}

In total this work reveals that both the DDR and telomere dysfunction are more prevalent in cells containing mitochondrial mutations. The severity of the effect however appears to related to the underlying mitochondrial mutation. This work suggests that damage to mtDNA may lead to increases in genomic instability and pave the way to a greater cancer risk. This work also highlights the fact that different qualities of radiation impact the biological results and predict that high LET radiation may be more damaging. Future more detailed studies monitoring ROS expression and its relationship to DDR and telomere length, as well as assaying for telomerase levels may help to better understand the mechanism linking defective mitochondria to these findings and the relationship to cancer formation.

\section{References}

1. Kadhim M, Salomaa S, Wright E, Hildebrandt G, Belyakov OV , et al. (2013) Nontargeted effects of ionising radiation--implications for low dose risk. Mut. Res 752: 84-98. [crossref]

2. Bartkova J, Horejsi Z, Koed K, Kramer A, Tort F, et al. (2005) DNA damage response as a candidate anti-cancer barrier in early human tumorigenesis. Nature 434: 864870. [crossref]

3. Lan Q, Cawthon R, Shen M, Weinstein SJ, Virtamo J, et al. (2009) A prospective study of telomere length measured by monochrome multiplex quantitative PCR and risk of non-Hodgkin lymphoma. Clinical cancer research an official journal of the American Association for Cancer Res 15: 7429-7433. [crossref]

4. Rowe LA, Degtyareva N, Doetsch PW (2008) DNA damage-induced reactive oxygen species (ROS) stress response in Saccharomyces cerevisiae. Free rad. biol. \& med 45: 1167-1177. [crossref]

5. Yang JL, Weissman L, Bohr VA, Mattson MP (2008) Mitochondrial DNA damage and repair in neurodegenerative disorders. DNA repair 7: 1110-1120. [crossref]

6. Hahn A, Zuryn S (2019) Mitochondrial Genome (mtDNA) Mutations that Generate Reactive Oxygen Species. Antioxidants 8. [crossref]

7. Yakes FM, Van Houten B (1997) Mitochondrial DNA damage is more extensive and persists longer than nuclear DNA damage in human cells following oxidative stress. PNAS 94: 514-519. [crossref]

8. Jakupciak JP, Maragh S, Markowitz ME, Greenberg AK, Hoque MO, et al. (2008) Performance of mitochondrial DNA mutations detecting early stage cancer. BMC cancer 8: 285. [crossref]

9. Sun N, Youle RJ, Finkel T (2016) The Mitochondrial Basis of Aging. Mol. Cell 61: 654-666. [crossref]

10. Brandon M, Baldi P, Wallace DC (2006) Mitochondrial mutations in cancer Oncogene 25: 4647-4662.

11. Nagasawa H, Little JB (1992) Induction of sister chromatid exchanges by extremely low doses of alpha-particles. Cancer res 52: 6394-6396. [crossref]

12. Ariyoshi K, Miura T, Kasai K, Fujishima Y, Nakata A, et al. (2019) Radiation-Induced Bystander Effect is Mediated by Mitochondrial DNA in Exosome-Like Vesicles. Scientific reports 9: 9103.

13. Trifunovic A, Wredenberg A, Falkenberg M, Spelbrink JN, Rovio AT, et al. (2004) Premature ageing in mice expressing defective mitochondrial DNA polymerase. Nature 429: 417-423

14. Kulkarni R, Marples B, Balasubramaniam M, Thomas RA, Tucker JD (2010) Mitochondrial gene expression changes in normal and mitochondrial mutant cells after exposure to ionizing radiation. Rad. Res 173: 635-644. [crossref]

15. Kulkarni R, Reither A, Thomas RA, Tucker JD (2009) Mitochondrial mutant cells are hypersensitive to ionizing radiation, phleomycin and mitomycin C. Mut. Res 663: 46-51. [crossref]

16. Sofou K, De Coo IF, Isohanni P, Ostergaard E, Naess K, et al. (2014) A multicenter study on Leigh syndrome disease course and predictors of survival. Orphanet journal of rare diseases 9: 52 . [crossref]

17. Yu-Wai-Man P, Griffiths PG, Hudson G, Chinnery PF (2009) Inherited mitochondrial optic neuropathies. Journal of medical genetics 46: 145-158. [crossref]

18. Noda A (2018) Radiation-induced unrepairable DSBs their role in the late effects of radiation and possible applications to biodosimetry. J. rad. Res 59. [crossref]

19. Bonner WM, Redon CE, Dickey IS, Nakamura AJ, Sedelnikova OA, et al. (2008) GammaH2AX and cancer. Nat. Rev. Cancer 8: 957-967. [crossref]

20. Gorgoulis VG, Pefani DE, Pateras IS, Trougakos IP (2018) Integrating the DNA damage and protein stress responses during cancer development and treatment. J. Pathol 246: 12-40. [crossref]

21. Kulkarni R, Thomas RA, Tucker JD (2011) Expression of DNA repair and apoptosis genes in mitochondrial mutant and normal cells following exposure to ionizing radiation. Environ. mol. Mutagen 52: 229-237. [crossref]

22. Deng Y, Chang S (2007) Role of telomeres and telomerase in genomic instability, senescence and cancer. Laboratory investigation a journal of technical methods and pathology 87: 1071-1076. [crossref] 
23. Wang Z, Zhang Z, Guo Y, Shui H, Liu G, et al. (2018) Shorter Telomere Length Is Associated with Increased Breast Cancer Risk in a Chinese Han Population A CaseControl Analysis. J. breast cancer 21: 391-398. [crossref]

24. Yamada-Hishida H, Nobeyama Y, Nakagawa H (2018) Correlation of telomere length to malignancy potential in non-melanoma skin cancers. Oncology let 15: 393-399. [crossref]

25. Jang JS, Choi YY, Lee WK, Choi JE, Cha SI, et al. (2008) Telomere length and the risk of lung cancer. Cancer science 99: 1385-1389. [crossref]

26. Yamada S, Misawa K, Mima M, Imai A, Mochizuki D, et al. (2021) Telomere shortening in head and neck cancer association between DNA demethylation and survival. J. Cancer 12: 2165-2172. [crossref]

27. Widmann TA, Herrmann M, Taha N, Konig J, Pfreundschuh M (2007) Short telomeres in aggressive non-Hodgkin's lymphoma as a risk factor in lymphomagenesis. Exp. Hem 35: 939-946.
28. McNally EJ, Luncsford PJ, Armanios M (2019) Long telomeres and cancer risk the price of cellular immortality. J. Clin. Invest 129: 3474-3481. [crossref]

29. Nan H, Du M, De Vivo I, Manson JE, Liu S, et al. (2011) Shorter telomeres associate with a reduced risk of melanoma development. Cancer res 71: 6758-6763. [crossref]

30. Burke LS, Hyland PL, Pfeiffer RM, Prescott J, Wheeler W, et al. (2013) Telomere length and the risk of cutaneous malignant melanoma in melanoma-prone families with and without CDKN2A mutations. PloS one 8: e71121. [crossref]

31. Xie H, Wu X, Wang S, Chang D, Pollock RE, et al. (2013) Long telomeres in peripheral blood leukocytes are associated with an increased risk of soft tissue sarcoma. Cancer 119: 1885-1891. [crossref]

32. Hackett JA, Greider CW (2002) Balancing instability dual roles for telomerase and telomere dysfunction in tumorigenesis. Oncogene 21: 619-626. [crossref]

33. Gutierrez-Rodrigues F, Santana-Lemos BA, Scheucher PS, Alves-Paiva RM, Calado RT (2014) Direct comparison of flow-FISH and qPCR as diagnostic tests for telomere length measurement in humans. PloS one 9: e113747. [crossref]

\section{Citation:}

Chen J, Sridharan D, Cross C, Pluth J (2021) Effects of Radiation on Mitochondrial Defective Lymphoblastoid Cells. Internal Med Res Open J Volume 6(4): 1-9. 